\title{
Differences in left ventricular functional adaptation to arterial stiffness and neurohormonal activation in patients with hypertension: a study with two-dimensional layer-specific speckle tracking echocardiography
}

Darae Kim, Chi Young Shim * (D), Geu-Ru Hong, Sungha Park, InJeong Cho, Hyuk-Jae Chang, Jong-Won Ha and Namsik Chung

\begin{abstract}
Background: Arterial stiffness increases pressure load to the left ventricle (LV), leading to LV hypertrophy and subendocardial ischemia. Neurohormones stimulate myocardial fibrosis and LV dysfunction. We aimed to explore the associations of arterial stiffness and plasma aldosterone with multi-directional, layer-specific LV, and left atrial (LA) mechanical function in hypertensive patients.

Methods: Layer-specific LV global longitudinal strain (GLS-trans, GLS-endo, GLS-epi), global circumferential strain (GCS-trans, GCS-endo, GCS-epi), LV torsional parameters, and LA global longitudinal strain (LA GLS) were analyzed by two-dimensional speckle tracking echocardiography in 195 hypertensive patients (110 men, mean age 55 years). Pulse wave velocity (PWV) was analyzed as a measure of arterial stiffness, and plasma aldosterone was measured for evaluation of neurohormonal activation.
\end{abstract}

Results: In a simple correlation, PWV significantly correlated with LV GLS-endo and LA GLS. Log aldosterone correlated with both LV GCS-endo and LV GCS-trans. Multiple regression analysis revealed that LV GLS-endo $(\beta=0.223, p=0.031)$ and LA GLS ( $\beta=-0.311, p=0.002$ ) were independently correlated with PWV even after controlling for confounding factors.

Conclusions: In hypertensive patients without clinically apparent target organ damage, LV GLS, especially endocardium, and LA GLS were more dominantly affected by arterial stiffness because, among the three myocardial layers, the endocardium is most susceptible to pressure overload. Two-dimensional layer-specific speckle-tracking echocardiography sensitively detects LV mechanical dysfunction and provides pathophysiologic insights into LV mechanical adaptations in hypertension.

Keywords: Left ventricle, Arterial stiffness, Aldosterone

\footnotetext{
* Correspondence: cysprs@yuhs.ac

Cardiology Division, Severance Cardiovascular Hospital, Yonsei University

College of Medicine, 50 Yonsei-ro, Seodaemun-gu, 120-752 Seoul, Republic

of Korea
} 


\section{Background}

Cardiac remodeling in hypertensive disease is a complex process involving both mechanical stress from central arterial stiffness and neurohormonal factors [1]. Arterial stiffness increases pressure load to the LV, increasing the size of myocytes and leading to LV hypertrophy. Neurohormones also play important roles in adverse remodeling of LV. Previous experimental studies revealed that aldosterone induced myocardial fibrosis, hypertrophy, and dysfunction [2, 3].

Progressive remodeling of LV is suggested to be heterogeneous across the LV wall [4]. The myocardium is composed of three myocardial layers; during the course of LV remodeling, central arterial stiffness and neurohormones can have different degrees of impact on the layers of the myocardium. Two-dimensional speckle tracking echocardiography provides quantitative information regarding LV deformation by longitudinal, circumferential, and torsion strain of myocardium [5]. Recently, layer-specific speckle-tracking echocardiography was reported to allow quantification of LV global longitudinal strain (GLS) and circumferential strain (GCS) of the three layers of the myocardium [6].

The aim of this study was to explore the associations of central arterial stiffness and plasma aldosterone with layer-specific LV and LA mechanical function in patients with early hypertension using two-dimensional speckle tracking echocardiography.

\section{Methods}

\section{Study subjects}

This study was designed as a retrospective cohort study. We included consecutive adult hypertensive patients without apparent end organ damage who underwent clinically indicated echocardiography between 2010 and 2015. Patients with reduced ejection fraction $(<50 \%)$, significant valvular heart disease, ischemic heart disease, idiopathic dilated cardiomyopathy, restrictive cardiomyopathy or hypertrophic cardiomyopathy, or atrial fibrillation were excluded from the study. Finally, a total of 195 patients were reviewed. Hypertension (HTN) was defined as systolic blood pressure (SBP) $\geq 140 \mathrm{mmHg}$, diastolic blood pressure (DBP) $\geq 90 \mathrm{mmHg}$, or current pharmacological treatment for HTN. Clinical data at the time of echocardiography were gathered from electronic medical records. Venous blood was collected in the morning after a 12-h fast with the patients in the sitting position to measure plasma aldosterone and renin on the same day as the echocardiography. Plasma aldosterone concentration (ng/dL) was measured by radioimmunoassay (Diagnostic Systems Laboratories Inc., TX). Plasma renin activity was measured by radioimmunoassay of angiotensin $I$ in the presence of reagents that inhibit angiotensin I-converting enzyme and angiotensinases. The assay was performed according to the method of Sealey using gammacoat plasma renin activity radioimmunoassay kits (DiaSorin [Stillwater, $\mathrm{MN}$ ], lower limit of determination is $0.1 \mathrm{ng} / \mathrm{mL} / \mathrm{h}$ ).

\section{Assessments of peripheral and central BPs}

Peripheral SBP and DBP measurements were performed automatically (Omron M4 Plus, Japan) at the brachial artery of the non-dominant arm in a relaxed seated position. Two BP measurements obtained at an interval of 5 min during the same visit were averaged. Central hemodynamics and parameters were assessed with pulse wave analysis of the radial artery using commercially available radial artery tonometry (SphygmoCor, AtCor Medical, Sydney, Australia) $[7,8]$. The measurements were obtained in the supine position after a minimum of 5 min of rest just before the echocardiogram. Peripheral pressure wave form was recorded from the radial artery with a high-fidelity micromanometer (Millar Instruments, Houston, TX) [8, 9]. Central systolic blood pressure (BP), diastolic BP, pulse pressure (PP), augmentation pressure, and augmentation index (AIx) were analyzed from 20 sequential pulse waveforms. PP was calculated as the difference between SBP and DBP. Augmentation pressure was the maximum systolic pressure minus pressure at the inflection point. The AIx was defined as AP divided by PP and expressed as a percentage. As in previous studies, because AIx is influenced by the heart rate, it was normalized for a heart rate of 75 bpm (AIx@75) [10]. Pulse wave velocity (PWV) was measured using carotid-femoral pressure pulse transit time.

\section{Two-dimensional and Doppler echocardiography}

Each patient underwent a comprehensive transthoracic echocardiographic study using a Vivid 7 or Vivid 9 cardiovascular ultrasound system (GE Medical Systems, Horten, Norway), equipped with 2.5-3.5 MHz phased-array sector probes. Standard 2D and Doppler measurements were performed according to the recommendations of the American Society of Echocardiography guidelines [11]. LV hypertrophy was diagnosed according to the American Society of Echocardiography recommended formula for estimation of LV mass index and was indexed to body surface area (cutoff values for LV mass index were $>115 \mathrm{~g} / \mathrm{m}^{2}$ for men and $>95 \mathrm{~g} / \mathrm{m}^{2}$ for women). From the apical window, mitral inflow velocities were traced, and the following variables were obtained: peak velocity of early diastolic mitral inflow (E), late diastolic mitral inflow (A), and deceleration time of the E velocity. Early diastolic mitral annular velocity (e'), late diastolic mitral annular velocity, and systolic mitral annular velocity $\left(\mathrm{S}^{\prime}\right)$ were measured from the apical fourchamber view with a 2- to 5-mm sample volume placed at the septal corner of the mitral annulus. 


\section{Two-dimensional speckle tracking strain analysis}

Three consecutive cardiac cycles were recorded and averaged, and the frame rates were set to 60-80 frames/s. The analysis was performed offline using customized software (EchoPAC PC, version 113; GE Medical Systems). The endocardial border of the LV was manually traced from three apical views (apical 4-, 2-, and 3chamber views) to obtain LV global longitudinal strain of endocardial (GLS-endo), transmural (GLS-trans), and epicardial strains (GLS-epi) by averaging all regional peak longitudinal strains. Layer-specific LV global circumferential strain (GCS) was obtained from averaging all circumferential strains from the short axis view of the mitral valve, papillary muscle, and LV apex level (LV GCS-endo, LV GCS-trans, LV GCS-epi). LV Torsion was automatically calculated as the instantaneous difference between LV apical and LV basal rotation.

The global longitudinal strain of the LA (LA-GLS) was measured by manually tracing the LA endocardial border in both four-chamber and two-chamber views. Because two segments of the LA roof demonstrated lower longitudinal strain curves than the other four, they were excluded from both 4-chamber and 2-chamber views. Therefore, global peak LALS during ventricular systole was measured by averaging the values obtained in the eight other LA segments. The time to peak LALS was also measured as the average of the eight segments and by calculating the time delay from the QRS to the positive peak LALS [12].

To examine intraobserver and interobserver variability for layer-specific LV GLS and GCS, the same observer and a second independent observer repeated the analysis of the first 10 consecutive patients. Intraobserver and interobserver intraclass coefficients were as follows: 0.97 (95\% confidence interval $[\mathrm{CI}]$ 0.96-0.98) and 0.96 (95\% CI 0.96-0.98) for LV GLS-endo; 0.96 (95\% CI 0.93-0.98) and 0.95 (95\% CI 0.90-0.99) for LV GLS-trans; 0.93 (95\% CI $0.78-0.96)$ and 0.89 (95\% CI 0.64-0.95) for LV GLS epi; 0.93 (95\% CI $0.90-0.98$ ) and 0.95 (95\% CI $0.92-0.98$ ) for LV GCS-endo; 0.96 (95\% CI 0.93-0.98) and 0.94 (95\% CI 0.89-0.98) for LV GCS-trans; 0.91 (95\% CI 0.75-0.96) and 0.88 (95\% CI $0.62-0.95)$ for LV GCS epi; 0.88 (95\% CI 0.84-0.92) and 0.90 (95\% CI 0.85-0.95) for LA GLS.

\section{Statistical analysis}

Continuous variables are presented as mean \pm SD and categorical variables are presented as absolute and relative frequencies (\%). Spearman's simple correlation analyses were performed to determine the associations between strain parameters and parameters of arterial stiffness and log aldosterone. Multiple linear regressions were performed to determine the independent association of LV mechanical parameters with arterial stiffness and neurohormone.

\section{Results}

Demographic characteristics

Table 1 shows baseline characteristics of our cohort. Mean age was $55 \pm 13$, and 96 patients (49\%) were men. Regarding medication, 108 (55\%) patients were taking angiotensin-converting enzyme inhibitors or angiotensin receptor blockers at the time of echocardiography. LV hypertrophy was present in $52(27 \%)$ patients. Table 2 shows echocardiographic findings in our cohort. Mean left atrial volume index $\left(24.5 \mathrm{ml} / \mathrm{m}^{2} \pm 7.9\right)$ and $\mathrm{E} / \mathrm{E}^{\prime}$ value $(10.4 \pm 3.5)$ were in the normal range. The mean value of LV GLS-trans was $-16.9 \pm 2.9$, which was slightly lower than the normal value [11].

\section{Central hemodynamics and neurohormone and the indices of $L V$ mechanical function}

Table 3 shows simple correlation analysis between strain parameters and PWV or log aldosterone. In a simple correlation, PWV was well correlated with LV GLS-endo $(r=0.169, p=0.046)$ and LA GLS $(r=-0.250, p=0.003)$.

Table 1 Baseline characteristics of the study population

\begin{tabular}{|c|c|}
\hline & $n=195$ \\
\hline \multicolumn{2}{|l|}{ Demographics } \\
\hline Age, years & $55 \pm 13$ \\
\hline Male gender, n (\%) & $96(49)$ \\
\hline Body mass index, $\mathrm{kg} / \mathrm{m}^{2}$ & $24.8 \pm 3.7$ \\
\hline Dyslipidemia, n (\%) & $50(26)$ \\
\hline Smoking, n (\%) & $21(11)$ \\
\hline \multicolumn{2}{|l|}{ Medications } \\
\hline RAAS blockers, n (\%) & $108(55)$ \\
\hline Beta blockers, n (\%) & $39(20)$ \\
\hline Calcium channel blockers, n (\%) & $68(35)$ \\
\hline Diuretics, n (\%) & $19(10)$ \\
\hline \multicolumn{2}{|l|}{ Hemodynamics } \\
\hline Peripheral SBP, mmHg & $148 \pm 76$ \\
\hline Peripheral DBP, mmHg & $83 \pm 13$ \\
\hline Peripheral PP, mmHg & $61 \pm 15$ \\
\hline Central SBP, mmHg & $132 \pm 18$ \\
\hline Central DBP, mmHg & $85 \pm 13$ \\
\hline Central PP, mmHg & $48 \pm 14$ \\
\hline Alx@75,\% & $24 \pm 12$ \\
\hline Heart rate, bpm & $68 \pm 12$ \\
\hline PP amplification & $1.31 \pm 0.21$ \\
\hline $\mathrm{PWV}, \mathrm{m} / \mathrm{s}$ & $8.3 \pm 1.8$ \\
\hline Aldosterone, ng/dL & $16.03(12.15-19.53)$ \\
\hline Renin, ng/mL/h & $1.55(0.68-3.71)$ \\
\hline
\end{tabular}

$R A A S$ renin angiotensin aldosterone system, $S B P$ systolic blood pressure, $D B P$ diastolic blood pressure, $P P$ pulse pressure, $A l x$ augmentation index, $P W V$ pulse wave velocity 
Table 2 Two-dimensional, Doppler, and speckle tracking echocardiography

\begin{tabular}{ll}
\hline & $n=195$ \\
\hline Two-dimensional and Doppler & \\
LV end-diastolic dimension, mm & $48 \pm 5$ \\
LV end-systolic dimension, mm & $31 \pm 4$ \\
IVS thickness, mm & $9.6 \pm 1.9$ \\
PW thickness, mm & $9.4 \pm 1.4$ \\
Relative wall thickness & $0.41 \pm 0.25$ \\
LV mass index, g/m ${ }^{2}$ & $92 \pm 25$ \\
LV hypertrophy, n (\%) & $52(27)$ \\
LV ejection fraction, \% & $67.9 \pm 5.8$ \\
$\mathrm{e}^{\prime}$ velocity, cm/s & $6.8 \pm 2.5$ \\
S' velocity, cm/s $^{\prime}$ & $7.4 \pm 1.5$ \\
E/e' & $10.4 \pm 3.5$ \\
LA volume index, ml/m² & $24.7 \pm 7.9$ \\
Two-dimensional speckle tracking & \\
LV GLS, \% & \\
Transmural & \\
Endocardial & $-16.9 \pm 2.9$ \\
Epicardial & $-19.1 \pm 3.3$ \\
LV GRS, \% & $-14.9 \pm 2.8$ \\
LV GCS, \% & $34.1 \pm 12.2$ \\
Transmural & \\
Endocardial & $-22.0 \pm 5.0$ \\
EVicardial & $31.7 \pm 9.1$ \\
LA GLS, \% & \\
\hline$V$ & \\
\hline
\end{tabular}

$L V$ left ventricle, $I V S$ interventricular septum, $P W$ posterior wall, $L A$ left atrium, GLS global longitudinal strain, GRS global radial strain,

GCS global circumferential strain, SD standard deviation

PWV showed significant correlation with LV GLS-endo even in patients without LV hypertrophy(Fig. 1). Other central hemodynamic parameters, such as PP amplification or AIx@ 75, did not show significant correlation with strain parameters. Log aldosterone showed positive correlation with LV GCS-endo $(r=0.251, p=0.019)$ and LV GCS-trans $(r=0.240, p=0.024)$, whereas no significant correlation was observed with LV GLS of any layer. Unlike the correlation between LV GLS-endo and PWV, log aldosterone did not show a significant correlation with LV GCS-endo in patients without LV hypertrophy (Fig. 2).

Multiple regression analysis revealed that LV GLSendo (beta $=0.223, p=0.031$ ) and LA GLS (beta $=-0.318$, $p=0.002)$ were independently correlated with PWV, even after controlling for confounding factors such as age, gender, body mass index, presence of diabetes
Table 3 Simple correlations

\begin{tabular}{|c|c|c|c|c|}
\hline & \multicolumn{2}{|l|}{ PWV } & \multicolumn{2}{|c|}{ Log Aldosterone } \\
\hline & $R$ & $P$-value & $\mathrm{R}$ & $P$-value \\
\hline \multicolumn{5}{|l|}{ LV GLS } \\
\hline Transmural & 0.145 & 0.090 & 0.062 & 0.511 \\
\hline Endocardial & 0.169 & 0.046 & 0.052 & 0.586 \\
\hline Epicardial & 0.162 & 0.056 & 0.056 & 0.552 \\
\hline LV GRS & 0.036 & 0.710 & -0.168 & 0.123 \\
\hline \multicolumn{5}{|l|}{ LV GCS } \\
\hline Transmural & -0.039 & 0.689 & 0.240 & 0.024 \\
\hline Endocardial & 0.016 & 0.870 & 0.251 & 0.019 \\
\hline Epicardial & -0.021 & 0.832 & 0.078 & 0.477 \\
\hline LV Torsion, peak & 0.026 & 0.786 & -0.139 & 0.191 \\
\hline LA GLS & -0.250 & 0.003 & 0.018 & 0.854 \\
\hline
\end{tabular}

mellitus, LV mass index, LV ejection fraction, and use of renin aldosterone angiotensin (RAAS) blockers (Table 4).

\section{Discussion}

This study presents correlation of LA and LA mechanical function with increased arterial stiffness and neurohormone level in patients with early hypertension without clinically apparent target organ damage. The principle finding from our results is that LV GLS, especially endocardium, and LA GLS were dominantly affected by central arterial stiffness in early hypertensive patients without clinically apparent target organ damage.

Two-dimensional speckling analysis provides sensitive assessment of mechanical function of LV and LA. Recently, LV GLS gained clinical significance in patients with hypertensive heart disease. Lee et al. showed that LV GLS, especially that of the epicardial layer, was the only independent predictor of events [13]. Saito et al. showed that LV GLS had additive predictive value to clinical risk factors and concentric LV hypertrophy for predicting adverse cardiovascular outcomes in asymptomatic hypertensive patients [14]. The mechanism for impaired longitudinal strain in hypertension has been studied recently. In an animal study, when LV GLS was compared with transmural histology of myocardium, fibrosis was predominant in the subendocardial layer, and longitudinal deformation of the LV was affected mainly by subendocardial fibrosis, whereas circumferential and radial strain were preserved until the late phase of the experiment [15]. Investigators demonstrated propagation of fibrosis from the subendocardium to the mid layer of the myocardium as HTN persisted, which affected radial strain in the late course. This finding is consistent with our data. From our data, PWV correlated 


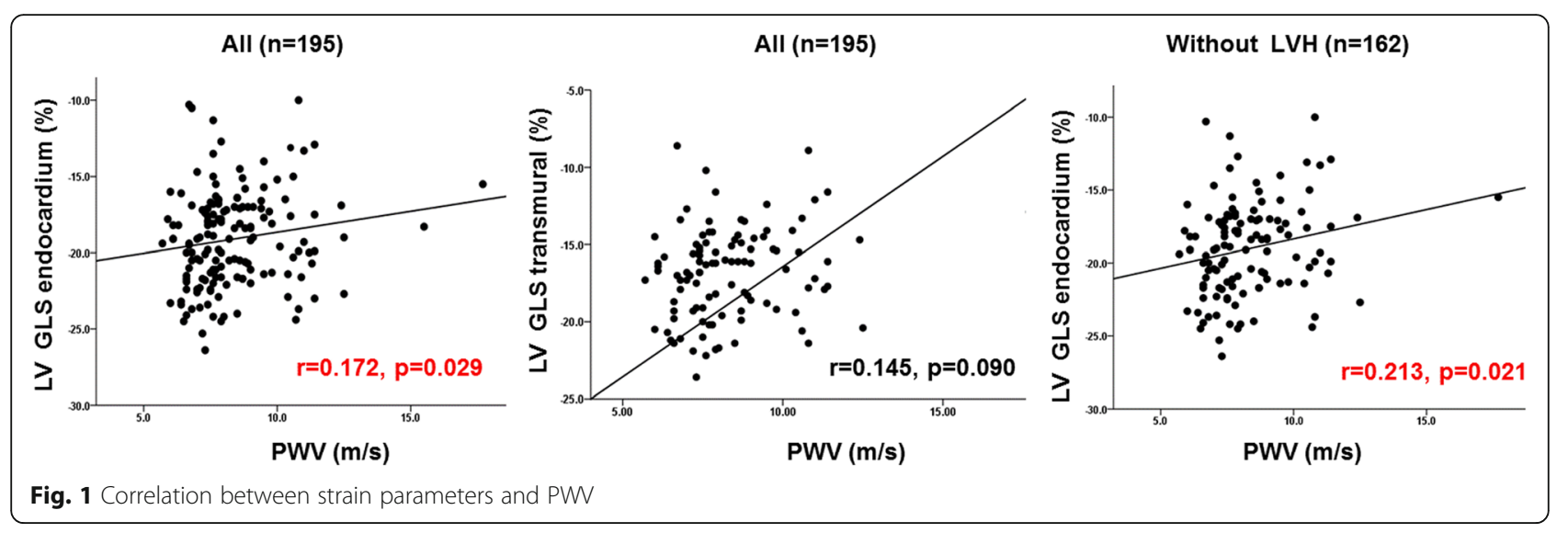

well with LV GLS-endo. The significance of the correlation between PWV and LV GLS-endo was significant even in patients without LV hypertrophy.

In addition, LA GLS showed independent correlation with PWV. Although current guidelines recommend assessment for presence of LV hypertrophy as a sign of target organ damage, increased LA size is strongly associated with the duration and level of systolic blood pressure [16]. Change of LA mechanical function and enlargement of LA can occur even before LV hypertrophy [17]. It is interesting that our data showed a significant correlation between LA GLS and PWV when most of patients did not have an enlarged LA, reflecting changes in mechanical function before structural adaptation. A previous study with a small number of patients showed reverse remodeling of LA with treatment of quinapril and independent correlation with improved central arterial stiffness [18]. Our finding suggests that LA GLS can serve as a sensitive surrogate marker for treatment response or risk stratification in early hypertensive patients who do not have enlarged LA or LV hypertrophy.
The renin-aldosterone system is reported to cause subendocardial fibrosis and stimulate hypertrophy of myocytes [19, 20]. In our study, log aldosterone showed significant correlation with LV GCS-endo and LV GCS-trans from simple correlation analysis, whereas no significant correlation was observed between LV GLS and log aldosterone. The correlation between LV GCS and log aldosterone was not significant in patients without LV hypertrophy. Although this finding cannot be fully explained due to the crosssectional nature of our study, the data suggest that neurohormonal factors affect myocardium in a different manner from mechanical stress due to increased arterial stiffness. A previous animal study suggested that circumferential strain is affected by later stages of HTN, as progressive fibrosis affects the middle layer of the myocardium. In our study, the correlation between LV GCS-endo and log aldosterone failed to show significance in multivariate analysis. This might reflect the characteristics of our study cohort. Many of the patients in our study were in early stages of hypertension, and 61 (31\%) had undetectable plasma aldosterone

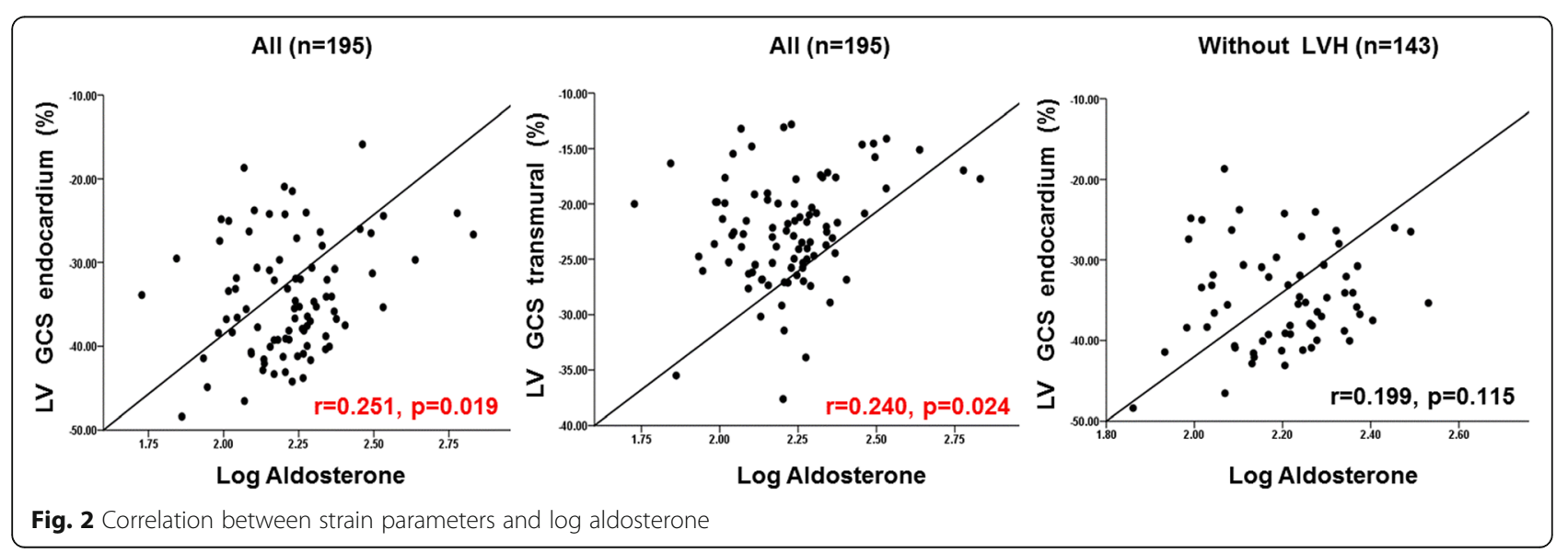


Table 4 Determinants for myocardial mechanical function

\begin{tabular}{|c|c|c|c|}
\hline & $\beta$ & $\mathrm{t}$ & $p$-value \\
\hline \multicolumn{4}{|c|}{ LV GLS endocardium $\left(R^{2}=0.327\right)$} \\
\hline Age & -0.170 & -1.48 & 0.142 \\
\hline Female gender & -0.112 & -1.22 & 0.225 \\
\hline Body mass index & 0.046 & 0.49 & 0.626 \\
\hline Diabetes mellitus & 0.062 & 0.66 & 0.511 \\
\hline Peripheral SBP & 0.024 & 0.26 & 0.795 \\
\hline LV ejection fraction & -0.419 & -4.44 & $<0.001$ \\
\hline LV mass index & -0.001 & -0.01 & 0.995 \\
\hline Use of RAAS blocker & 0.158 & 1.76 & 0.082 \\
\hline PW & 0.223 & 2.19 & 0.031 \\
\hline \multicolumn{4}{|c|}{ LV GCS endocardium $\left(R^{2}=0.164\right)$} \\
\hline Age & 0.190 & 1.21 & 0.231 \\
\hline Female gender & -0.130 & -1.06 & 0.294 \\
\hline Body mass index & 0.076 & 0.63 & 0.534 \\
\hline Diabetes mellitus & 0.108 & 0.87 & 0.385 \\
\hline Peripheral SBP & 0.004 & 0.04 & 0.970 \\
\hline LV ejection fraction & -0.293 & -2.35 & 0.022 \\
\hline LV mass index & -0.015 & -0.13 & 0.896 \\
\hline Use of RAAS blocker & -0.091 & -0.76 & 0.448 \\
\hline Log Aldosterone & 0.201 & 1.61 & 0.112 \\
\hline \multicolumn{4}{|l|}{ LA GLS $\left(R^{2}=0.361\right)$} \\
\hline Age & -0.118 & -1.05 & 0.297 \\
\hline Female gender & -0.096 & -1.06 & 0.292 \\
\hline Body mass index & -0.179 & -1.94 & 0.055 \\
\hline Diabetes mellitus & -0.158 & -1.70 & 0.092 \\
\hline Peripheral SBP & -0.044 & -0.50 & 0.622 \\
\hline LV ejection fraction & 0.218 & 2.35 & 0.021 \\
\hline LV mass index & -0.065 & -0.74 & 0.461 \\
\hline Use of RAAS blocker & -0.318 & -3.59 & 0.001 \\
\hline PWV & -0.311 & -3.12 & 0.002 \\
\hline
\end{tabular}

$S B P$ systolic blood pressure, $L V$ left ventricle, $R A A S$ renin angiotensin aldosterone system, $P W V$ pulse wave velocity and renin. The correlation between neurohormone and LV mechanics should be studied in a large number of patients with more advanced hypertension.

Early detection of cardiac structural changes in early hypertension is important with regard to risk stratification. Based on the findings from our study, LV and LA remodeling in early hypertension can be hypothesized as outlined in Fig. 3. Mechanical stress from pressure overload impairs LV GLS of the endocardium and LA GLS. Neurohormones and aldosterone can impair LV GCS of the endocardium and transmural myocardium as hypertension progresses.

\section{Limitations}

Several limitations of this study need to be noted. First, we included patients with early hypertension. Patients with LV hypertrophy represented only $27 \%$ of our cohort, and the small number of patients with LV hypertrophy might have affected the correlation analysis of the LV hypertrophy subgroup. Second, $51 \%$ of our patients were on RAAS blockers, which might have affected reverse remodeling of LV. However, we included use of RAAS blocker in multivariate analysis to minimize confounding effects. Third, because of the cross-sectional design of our study, we could not determine whether treatment of HTN can reverse abnormal LV mechanics. All of these issues are relevant and should be examined in future studies.

\section{Conclusion}

Central arterial stiffness was independently correlated with LV GLS, especially endocardium, because the endocardium is the most susceptible to pressure overload among the three myocardial layers. Also, central arterial stiffness showed an independent negative correlation with LA GLS. Aldosterone showed significant positive correlation with LV GCS, especially endocardium from simple correlation, however, failed to remain significant in multivariate analysis. Different

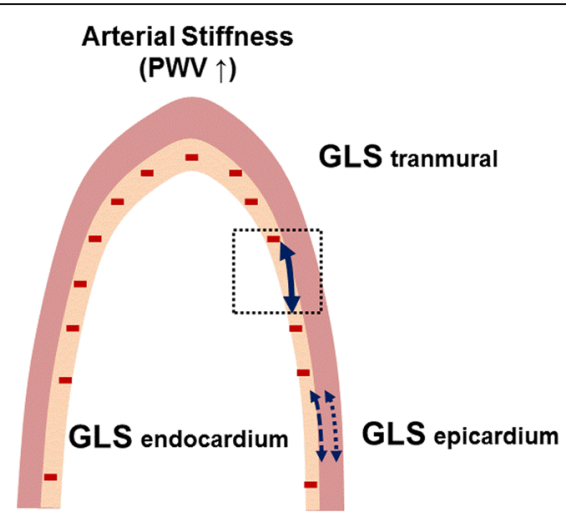

Neurohormonal Activation (Aldosterone $\uparrow$ )

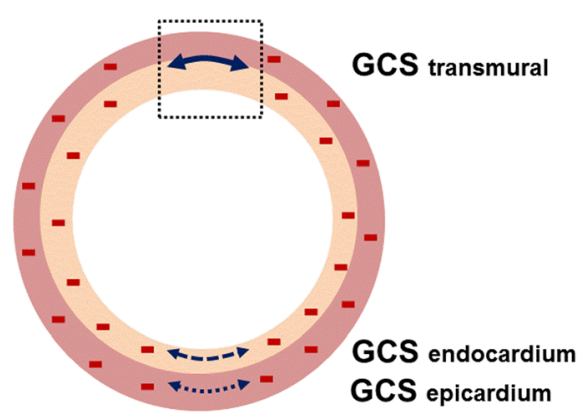

Fig. 3 Hypothesis on LV and LA remodeling in early hypertension 
correlations between strain parameters and central arterial stiffness or aldosterone suggest different functional adaptations of LV and LA to arterial stiffness and neurohormone. In addition, two-dimensional layerspecific speckle-tracking echocardiography sensitively detected LV and LA mechanical adaptation in early hypertension, even without LV hypertrophy, and provided pathophysiologic insights into LV and LA remodeling in association with central stiffness.

\begin{abstract}
Abbreviations
Alx: Augmentation index; BP: Blood pressure; DBP: Diastolic blood pressure; GCS: Global circumferential strain; GLS: Global longitudinal strain; GLSendo: Endocardial global circumferential strain; GLS-endo: Endocardial global longitudinal strain; GLS-epi: Epicardial global circumferential strain; GLSepi: Epicardial global longitudinal strain; GLS-trans: Transmyocardial global circumferential strain; GLS-trans: Transmyocardial global longitudinal strain; HTN: Hypertension; LA: Left atrium; LV: Left ventricle; PP : Pulse pressure; PWV: Pulse wave velocity; RAAS: Renin angiotensin aldosterone system; SBP: Systolic blood pressure; SD: Standard deviation
\end{abstract}

\section{Acknowledgements}

Not applicable

\section{Funding}

This study was supported by the grant "Research grant from Korean Society of Hypertension, 2014".

\section{Availability of data and materials}

Raw data can be made available on reasonable requests to the corresponding author.

\section{Authors' contributions}

$\mathrm{KD}$ and SCY participated in the study design, selection of study subjects, statistical analysis and helped to draft the manuscript. HGR, PS, CIJ, CHJ, HJW, and $\mathrm{CN}$ participated in its design and coordination. All authors read and approved the final manuscript.

\section{Ethics approval and consent to participate}

The study was approved by the institutional review board of Severance hospital. All patients provided written informed consent.

\section{Consent for publication}

Not applicable. No individual data in any form is disclosed.

\section{Competing interests}

The authors declare that they have no competing interests for this study.

\section{Publisher's Note}

Springer Nature remains neutral with regard to jurisdictional claims in published maps and institutional affiliations.

Received: 7 August 2017 Accepted: 27 September 2017

Published online: 02 November 2017

\section{References}

1. Drazner MH. The progression of hypertensive heart disease. Circulation. 2011;123(3):327-34.

2. Weber KT, Brilla CG. Pathological hypertrophy and cardiac interstitium. Fibrosis and renin-angiotensin-aldosterone system. Circulation. 1991;83(6): 1849-65.

3. Lacolley P, Safar ME, Lucet B, Ledudal K, Labat C, Benetos A. Prevention of aortic and cardiac fibrosis by spironolactone in old normotensive rats. J Am Coll Cardiol. 2001;37(2):662-7.

4. Kuwada Y, Takenaka K. Transmural heterogeneity of the left ventricular wall: subendocardial layer and subepicardial layer. J Cardiol. 2000;35(3):205-18.

5. Geyer H, Caracciolo G, Abe H, Wilansky S, Carerj S, Gentile F, et al. Assessment of myocardial mechanics using speckle tracking echocardiography: fundamentals and clinical applications. J Am Soc Echocardiogr. 2010;23(4):351-69. quiz 453-5

6. Leitman M, Lysiansky M, Lysyansky P, Friedman Z, Tyomkin V, Fuchs T, et al. Circumferential and longitudinal strain in 3 myocardial layers in normal subjects and in patients with regional left ventricular dysfunction. J Am Soc Echocardiogr. 2010;23(1):64-70.

7. Weber T, O'Rourke MF, Ammer M, Kvas E, Punzengruber C, Eber B. Arterial stiffness and arterial wave reflections are associated with systolic and diastolic function in patients with normal ejection fraction. Am J Hypertens. 2008;21(11):1194-202.

8. Weber T, Auer J, O'Rourke MF, Kvas E, Lassnig E, Berent R, et al. Arterial stiffness, wave reflections, and the risk of coronary artery disease. Circulation. 2004;109(2):184-9.

9. Shim CY, Park S, Choi D, Yang WI, Cho IJ, Choi EY, et al. Sex differences in central hemodynamics and their relationship to left ventricular diastolic function. J Am Coll Cardiol. 2011;57(10):1226-33.

10. Wilkinson IB, MacCallum H, Flint L, Cockcroft JR, Newby DE, Webb DJ. The influence of heart rate on augmentation index and central arterial pressure in humans. J Physiol 2000;525(1):263-70.

11. Lang RM, Badano LP, Mor-Avi V, Afilalo J, Armstrong A, Ernande L, et al. Recommendations for cardiac chamber quantification by echocardiography in adults: an update from the American Society of Echocardiography and the European Association of Cardiovascular Imaging. Eur Heart J Cardiovasc Imaging. 2015;16(3):233-70.

12. Kim D, Shim CY, Hong GR, Kim MH, Seo J, Cho IJ, et al. Clinical Implications and Determinants of Left Atrial Mechanical Dysfunction in Patients With Stroke. Stroke. 2016;47(6):1444-51.

13. Lee WH, Liu YW, Yang LT, Tsai WC. Prognostic value of longitudinal strain of subepicardial myocardium in patients with hypertension. J Hypertens. 2016; 34(6):1195-200

14. Saito M, Khan F, Stoklosa T, lannaccone A, Negishi K, Marwick TH. Prognostic Implications of LV Strain Risk Score in Asymptomatic Patients With Hypertensive Heart Disease. JACC Cardiovasc Imaging. 2016;9(8):911-21.

15. Ishizu T, Seo Y, Kameda Y, Kawamura R, Kimura T, Shimojo N, et al. Left ventricular strain and transmural distribution of structural remodeling in hypertensive heart disease. Hypertension. 2014;63(3):500-6.

16. Vaziri SM, Larson MG, Lauer MS, Benjamin EJ, Levy D. Influence of blood pressure on left atrial size. The Framingham Heart Study. Hypertension. 1995;25(6):1155-60.

17. Aljizeeri A, Gin K, Barnes ME, Lee PK, Nair P, Jue J, et al. Atrial remodeling in newly diagnosed drug-naive hypertensive subjects. Echocardiography. 2013; 30(6):627-33.

18. Tsang TS, Barnes ME, Abhayaratna WP, Cha SS, Gersh BJ, Langins AP, et al. Effects of quinapril on left atrial structural remodeling and arterial stiffness. Am J Cardiol. 2006;97(6):916-20.

19. Karam H, Heudes D, Hess P, Gonzales MF, Loffler BM, Clozel M, et al. Respective role of humoral factors and blood pressure in cardiac remodeling of DOCA hypertensive rats. Cardiovasc Res. 1996;31(2):287-95.

20. Okoshi MP, Yan X, Okoshi K, Nakayama M, Schuldt AJ, O'Connell TD, et al. Aldosterone directly stimulates cardiac myocyte hypertrophy. J Card Fail. 2004;10(6):511-8.
Submit your next manuscript to BioMed Central and we will help you at every step:

- We accept pre-submission inquiries

- Our selector tool helps you to find the most relevant journal

- We provide round the clock customer support

- Convenient online submission

- Thorough peer review

- Inclusion in PubMed and all major indexing services

- Maximum visibility for your research

Submit your manuscript at www.biomedcentral.com/submit
Ciomed Central 